\title{
Análisis retrospectivo de las características y utilización de los recursos de salud en pacientes con cáncer de próstata resistente a la castración metastásico tratados con Enzalutamida o Acetato de Abiraterona en Colombia
}

\section{Retrospective Analysis of the Characteristics and Utilization of Health Resources in Patients with Castration Resistant Prostate Cancer Treated with Enzalutamide or Abiraterone Acetate in Colombia}

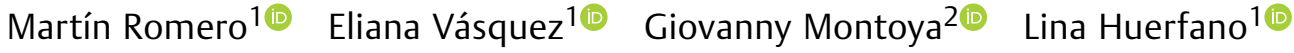 \\ ${ }^{1}$ Grupo de investigación ProyectaMe, Bogota, Colombia \\ ${ }^{2}$ Astellas Farma Colombia, Bogota, Colombia \\ Address for correspondence Martín Romero, Grupo de investigación \\ ProyectaMe, Bogotá, Colombia \\ (e-mail: martin.romero@proyecta-me.com).
}

Urol Colomb 2021;30:15-22.

\section{Resumen}

\section{Palabras claves}

- neoplasias de la próstata

- castración

- metástasis de la neoplasia

- antagonistas de hormonas

- costos de la atención en salud

- sistemas de salud
Objetivo Describir las características demográficas, clínicas, los recursos de salud utilizados y los costos directos del tratamiento del cáncer de próstata resistente a la castración metastásico (CPRCm), tratados con enzalutamida o acetato de abiraterona en Colombia.

Métodos Mediante un estudio de corte transversal se analizaron datos de utilización de servicios de una EPS (entidad promotora de salud) colombiana. Para ello, se identificaron los pacientes que durante el 1 de julio de 2016 y 30 de junio de 2017 (fecha índice) presentaron registro de uso de enzalutamida o acetato de abiraterona y se confirmaron sus antecedentes de castración química o quirúrgica (6 meses previos a la fecha índice). Se establecieron las frecuencias de uso de servicios en dicha población y se extrajo el consumo reportado asociado a consultas (urgencias, consulta externa), procedimientos y medicamentos en el periodo de análisis. Los costos se organizaron por categorías y se reportaron en pesos colombianos (COP) 2016.

Resultados se identificaron 161 pacientes con CPRCm con edad media de 77 años, se determinó que el departamento colombiano con más población de $\mathrm{CPRCm}$ en esta cohorte fue el Valle del Cauca, que 98,1\% de los pacientes pertenecen al régimen contributivo y que $1,9 \%$ al subsidiado. El cálculo de la supervivencia con el índice de comorbilidad de Charlson mostró que esos pacientes tendrían una tasa de received

March 4, 2020

accepted

June 17, 2020

published online

November 3, 2020
DOI https://doi.org/

$10.1055 / \mathrm{s}-0040-1714725$.

ISSN 0120-789X.

e ISSN 2027-0119.
(C) 2020. Sociedad Colombiana de Urología. All rights reserved. This is an open access article published by Thieme under the terms of the Creative Commons Attribution-NonDerivative-NonCommercial-License, permitting copying and reproduction so long as the original work is given appropriate credit. Contents may not be used for commercial purposes, or adapted, remixed, transformed or built upon. (https://creativecommons.org/ licenses/by-nc-nd/4.0/)

Thieme Revinter Publicações Ltda., Rua do Matoso 170, Rio de Janeiro, RJ, CEP 20270-135, Brazil 


\begin{abstract}
Keywords

- prostatic neoplasms

- castration

- neoplasm metástasis

- hormone antagonists

- health care costs

- health systems

Objective describe the demographic, clinical characteristics, the health resources utilization and the direct costs of patients with metastatic castration-resistant prostate cancer $(\mathrm{CPRCm})$ treated with Enzalutamide or Abiraterone Acetate in Colombia.

Methods Through a descriptive retrospective analysis through the database of a Colombian health insurer, patients were identified who, during July $1^{\text {st }}, 2016$ and June $30^{\text {th }}, 2017$ (index date) were dispensed with enzalutamide or abiraterone acetate, medical history of chemical or surgical castration was confirmed ( 6 months prior to the index date). The frequencies of use of services in this population were established and the reported consumption associated with consultations (emergencies, outpatient consultation), procedures and medications in the period of analysis were extracted. The costs were organized by categories and were reported according to Colombian pesos (COP) 2016.

Results In a cohort of 161 patients with CPRCm with an average age of 77 years, it was determined that Valle del Cauca is the Colombian department with the largest CPRCm population in this cohort. $98.1 \%$ of the patients belong to the contributory regimen and $1.9 \%$ to the subsidized. The calculation of the patient's survival with the Charlson Comorbidity Index $(\mathrm{CCl})$ showed a $0 \%$ survival rate at 10 years and most frequent comorbidities were hypertension, diabetes mellitus, renal failure and other related tumor pathologies. The total cost of managing a patient with $C P R C m$, who receives continuous abiraterone or enzalutamide for 12 months on average is $\$ 131,942,292$ COP per year, this figure includes both the use of services and the consumption of medications; the latter represents $\sim 90 \%$ of cost.

Conclusions These patients were characterized by being large demander of health services, namely, medicines, laboratories, images, outpatient visits and inpatient admission. Due to it is a small sample of patients, is necessary to develop a posterior evaluation to corroborate the findings found in this retrospective analysis.
\end{abstract}

supervivencia de $0 \%$ a 10 años. Las comorbilidades más frecuentes encontradas fueron hipertensión, diabetes mellitus, insuficiencia renal y otras patologías tumorales relacionadas. El costo total de manejo de un paciente con $\mathrm{CPRCm}$, que recibe de manera continua durante 12 meses abiraterona o enzalutamida es promedio $\$ 131.942 .292$ COP año. Esa cifra incluye tanto el uso de servicios como el consumo de medicamentos, y ese último representa cerca del 90\% del costo.

Conclusiones los pacientes con cáncer en estadio metastásico se caracterizan por presentar alta demanda de medicamentos, laboratorios, imágenes diagnósticas, visitas ambulatorias e ingresos hospitalarios. Debido a que es una muestra pequeña de pacientes, se requiere desarrollar una valoración posterior que permita corroborar los hallazgos encontrados en este estudio retrospectivo.

\section{Introducción}

Para el año 2012, en el mundo había un estimado de 1,1 millones de hombres con cáncer de próstata, representando el $15 \%$ de todos los diagnósticos de cáncer en hombres y de ellos, el 70\% se encontraban en las regiones más desarrolladas. ${ }^{1}$ En Colombia, para el año 2016 el cáncer de próstata presentaba una prevalencia de 87 casos por cada 100,000 hombres, ${ }^{2}$ siendo la primera causa de cáncer en hombres y la segunda causa de muerte. El cuadro clínico de esa enfermedad inicia con una progresión local, seguido de una diseminación hacia los ganglios linfáticos que más tarde puede conducir a metástasis óseas y viscerales. ${ }^{3}$ A pesar de los tratamientos hormonales usados, cerca del $80 \%$ presenta progresión dando lugar al cáncer de próstata metastásico resistente a castración $(\mathrm{CPRCm}),{ }^{4}$ el cual es definido como el aumento progresivo del antígeno prostático (PSA), acompañado de lesiones en ganglios o en vísceras presentando un aumento mesurable de la enfermedad, pese a presentar niveles de testosterona por debajo de $50 \mathrm{ng} / \mathrm{ml} \mathrm{(.4,5}$ Él por su parte, representa entre el 10-20\% de todos los casos de cáncer de próstata, y se caracteriza por tener un mal pronóstico, debido a que se asocia con una tasa de muerte considerablemente alta con una supervivencia que oscila entre los 18 y 24 meses. $^{4}$ 
Actualmente, y para este estadio de la enfermedad, se ha dado la aprobación del uso de dos agentes hormonales de segunda generación, considerados como las principales estrategias de tratamiento, para pacientes previamente manejados con quimioterapia o no. La enzalutamida es un inhibidor oral de la señal de los receptores androgénicos, y se usa en monoterapia, en administración una vez al día de $160 \mathrm{mg}^{6}$ y el acetato de abiraterona inhibidor de la biosíntesis de andrógenos, ${ }^{6}$ junto a la prednisolona para la administración oral una vez al día en dosis de $1000 \mathrm{mg}$ y $10 \mathrm{mg}$, respectivamente. ${ }^{7}$ Esos medicamentos están disponibles para su comercialización en el país, sin embargo, no se encuentran incluidos en el PBS (plan básico de salud). En la actualidad, los pacientes pueden acceder a esas tecnologías por el sistema de recobros. $^{8}$

No se han realizado estudios más allá del costo farmacológico, ${ }^{9}$ que permitan determinar la carga económica de la atención integral de dichos pacientes para el sistema de salud. Por lo tanto, resulta relevante un análisis de costos en la vida real de una cohorte de pacientes con $\mathrm{CPRCm}$, como una aproximación a lo que serían los costos globales de la atención de esa condición en Colombia.

\section{Métodos}

Se realizó un estudio retrospectivo con dos componentes, el primero, la descripción de las características de los pacientes con $\mathrm{CPRCm}$; el segundo, un análisis de los costos directos derivados de la atención de esa patología. El estudio partió de una base de datos de utilización de servicios de una EPS que cuenta con cerca de 3,2 millones de afiliados en todo el país, sobre la cual se identificaron a los pacientes mayores de 18 años con diagnóstico de cáncer de próstata de acuerdo con los códigos de diagnóstico CIE -10: C61x y D075. ${ }^{10}$ Posteriormente, se determinó la forma de identificación del estadio (- Figura 1), se consideraron resistentes a la castración y en fase metastásica quienes contaron con registro de castración quirúrgica (orquiectomía) o química (mínimo una dosis de hormona liberadora de hormona luteinizante - LHRH); y con registro de dispensación de enzalutamida o acetato de abiraterona entre el 1 de julio de 2016 y el 30 de junio de 2017. Ese algoritmo de selección fue validado por un experto (urólogo/oncólogo)

Las características iniciales analizadas incluyeron la edad del paciente, la localización geográfica, el régimen de afiliación en salud, las comorbilidades y los tratamientos recibidos relacionados con la enfermedad. Como desenlaces secundarios se estimaron las frecuencias de uso de servicios durante 12 meses, en las siguientes categorías: consulta externa, exámenes de laboratorio, imágenes, consulta por urgencias (pacientes admitidos y no admitidos para hospitalización) y días de estancia hospitalaria. Como desenlace exploratorio se calculó la sobrevida a 10 años por medio del índice de comorbilidad de Charlson, el cual toma en cuenta la edad el paciente y la presencia de determinadas comorbilidades para estimar la esperanza de vida. ${ }^{11}$

Se estableció como fecha índice, el registro del primer tratamiento en los pacientes con CPRCm con enzalutamida o acetato de abiraterona entre el 1 de julio de 2016 y el 30 de junio de 2017. Se analizaron los reportes de los seis meses previos a la fecha índice con el fin de identificar el tipo de castración que presentaban los pacientes, denominándose periodo pre-índice; así como los 6, 9 y 12 meses posteriores a la fecha índice, con el fin de analizar costos de recursos y la supervivencia (Diseño del

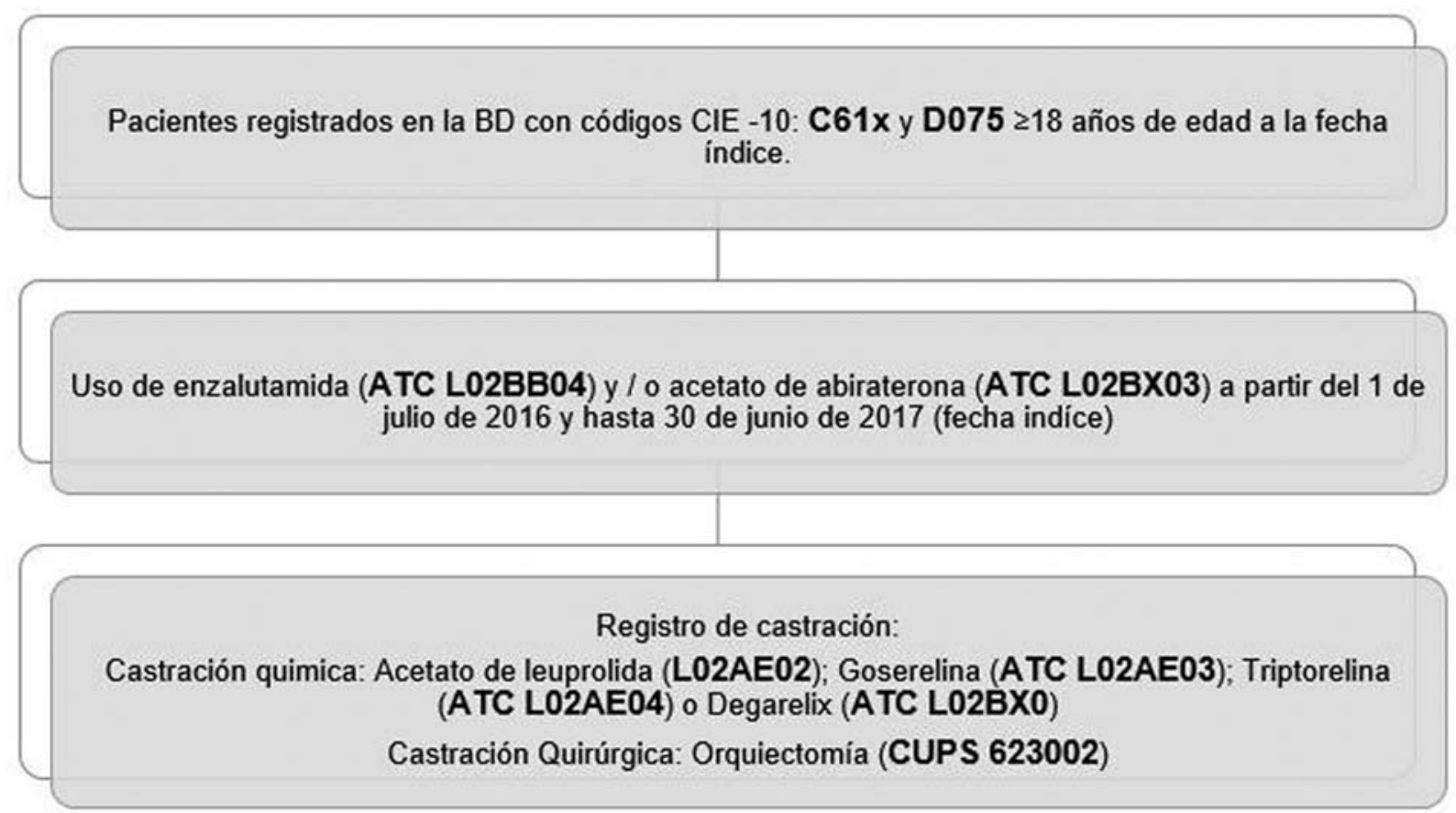

Fig. 1 Diagrama de identificación y selección de pacientes para el análisis. ATC: siglas en ingles de Anatomical Therapeutic Chemical Classification System; CUPS: Clasificación Única de Procedimientos en Salud en Colombia. Fuente: elaborado por los autores, 2019. 


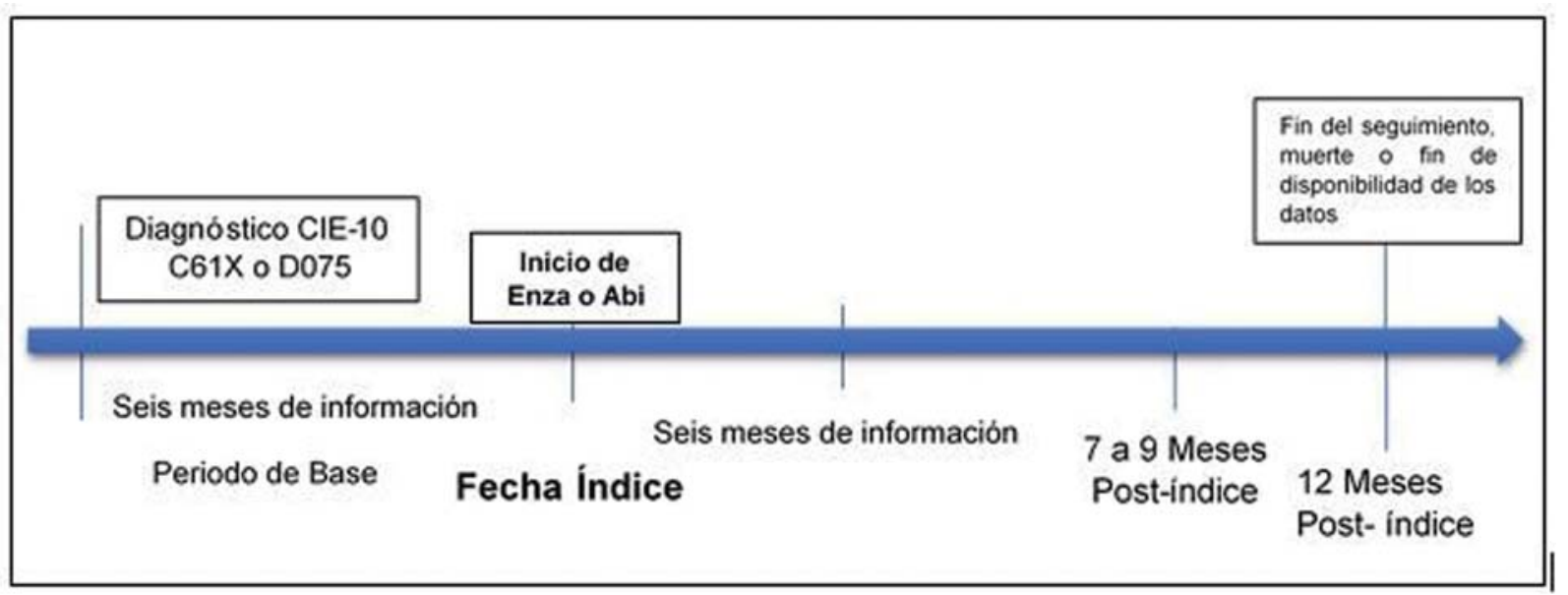

Fig. 2 Diseño del estudio. Fuente: elaborado por los autores, 2019.

estudio; - Figura 2), todo el proceso estuvo a cargo de los investigadores junto con el acompañamiento de expertos temáticos (urólogos y oncólogos).

Una vez identificados los pacientes con los criterios de inclusión, se extrajeron los consumos reportados por cada uno de ellos, y se clasificaron en los grupos: consulta externa, exámenes de laboratorio, imágenes, consulta por urgencias (pacientes hospitalizados y no admitidos para hospitalización) y días de estancia hospitalaria. Se incluyeron para el cálculo del costo medio de atención aquellos pacientes con consumo consecutivo durante 12 meses de enzalutamida o acetato de abiraterona y aquellos que no completaron los 12 meses por evento de muerte. Adicionalmente, se desarrolló un su análisis del uso de servicios y costos en los pacientes que presentaron o no tratamiento previo con quimioterapia. Los resultados se presentan en pesos colombianos 2016.

Para el análisis estadístico de los datos se usó la media y desviación estándar para las variables continuas y proporciones para las variables categóricas. De igual manera, se analizó la información de los pacientes con la prueba estadística TStudent en el caso de que la población para los grupos establecidos fuera $\mathrm{n}<30$ y con una distribución estadística normal en el caso de $\mathrm{n}>30$, estableciendo los respectivos intervalos de confianza del $95 \%$ para dar mayor validez estadística.

\section{Resultados}

\section{Características de los Pacientes}

Se identificaron un total de 3143 pacientes con diagnóstico de cáncer de próstata (C61X o D075) de una población masculina de $1.401,492$ (0,22\%). De esos pacientes, se identificaron 161 pacientes con CPRCm; 15 presentaron registro de dispensación de enzalutamida (9,3\%), 129 acetato de abiraterona $(80,1 \%)$ y 17 pacientes presentaron terapia secuencial con ambos medicamentos (10,6\%). De los 161 pacientes identificados, $152(94,4 \%)$ tenían registro de castración química, y 9 (5,6\%) castración quirúrgica.

La edad media de todos los pacientes identificados fue 77 años $\pm 11,33$, la edad media de los pacientes tratados con enzalutamida fue 74 años $\pm 11,11$ y la edad media para los pacientes con acetato de abiraterona fue 77 años $\pm 11,72$, mientras que para la terapia combinada fue de 80 años \pm 7,12. La - Tabla 1 muestra las características basales y demográficas de la población.

La - Tabla 2 muestra las comorbilidades discriminadas por grupo de edad.

\section{Uso de Servicios}

El servicio con mayor demanda fue el laboratorio clínico. Se puede evidenciar que un paciente, independiente de la tecnología, utiliza ese servicio 3 veces al mes en promedio; seguido de la consulta externa que en promedio la utiliza 2 veces al mes. Se estimó que los pacientes con uso de enzalutamida requieren 0,06 consultas de urgencias en promedio al mes, misma frecuencia que los pacientes con abiraterona. En cuanto a las imágenes, se observó una mayor demanda en el grupo de pacientes con acetato de abiraterona con 0,34 servicios mensuales ( 4 anuales) frente a 0,15 en el grupo de enzalutamida ( 2 anuales). El uso del servicio de hospitalización se mantuvo menor en el grupo de enzalutamida con una diferencia de un 33\% en el número de días de estancia hospitalaria. En la - Tabla 3 se observa la distribución de los servicios por grupo.

El análisis de los datos permitió identificar que 37 pacientes han recibido quimioterapia, 19 de ellos $(51,44 \%)$ la recibieron previo al uso de enzalutamida o acetato de abiraterona y 18 $(48,6 \%)$ recibieron el tratamiento con quimioterapia posterior al consumo de los medicamentos de interés.

Por otra parte, se pudo establecer dentro del seguimiento, un total de 45 muertes dentro de los pacientes analizados, determinándose una tasa de muerte general del $28 \%$. En el grupo de pacientes con enzalutamida se registraron 3 muertes, 6 en el grupo de terapia secuencial y 36 en los pacientes con acetato de abiraterona, correspondientes a tasas de muerte de 20\%, 27,9\% y $35,3 \%$, respectivamente.

\section{Costos}

Se encontró que el costo del tratamiento continuo por 12 meses con alguna de las dos tecnologías es en promedio \$131.942,292 
Tabla 1 Características basales y demográficas de la población

\begin{tabular}{|c|c|c|c|}
\hline Características de la población $(n=161)$ & $\begin{array}{l}\text { Enzalutamida } \\
n=15(9,3 \%)\end{array}$ & $\begin{array}{l}\text { Acetato de } \\
\text { abiraterona } \\
n=129(80,1 \%)\end{array}$ & $\begin{array}{l}\text { Terapia } \\
\text { secuencial } \\
n=17(10,6 \%)\end{array}$ \\
\hline \multicolumn{4}{|l|}{ Edad, media $\pm \mathrm{DE}$} \\
\hline Grupos de edad & $74 \pm 11,11$ & $77 \pm 11,72$ & $80 \pm 7,12$ \\
\hline $50-59(\%)$ & $2(13,3 \%)$ & $7(5,4 \%)$ & $0(0 \%)$ \\
\hline $60-69(\%)$ & $4(26,7 \%)$ & $27(20,9 \%)$ & $1(5,9 \%)$ \\
\hline $70-79(\%)$ & $4(26,7 \%)$ & $59(45,7 \%)$ & $7(41,2 \%)$ \\
\hline$>80(\%)$ & $5(33,3 \%)$ & $36(27,9 \%)$ & $9(52,9 \%)$ \\
\hline \multicolumn{4}{|l|}{ Localización geográfica } \\
\hline Región Andina, n (\%) & $7(46,7)$ & $65(51,6)^{*}$ & $3(17,6)$ \\
\hline Región Caribe, n (\%) & $5(33,3)$ & $24(19)$ & $5(29,4)$ \\
\hline Región Pacífica, n (\%) & $3(20)$ & $37(29,4)$ & $9(52,9)$ \\
\hline \multicolumn{4}{|l|}{ Régimen de afiliación } \\
\hline Contributivo, n (\%) & $15(100)$ & $126(97,7)$ & $17(100)$ \\
\hline Subsidiado, n (\%) & & $3(2,3)$ & \\
\hline \multicolumn{4}{|l|}{ Comorbilidades $^{* *}$} \\
\hline Hipertensión esencial (primaria) & $12(80 \%)$ & $91(70,5 \%)$ & $12(70,6 \%)$ \\
\hline Diabetes insulino dependiente & $4(26,7 \%)$ & $45(34,9 \%)$ & $4(23,5 \%)$ \\
\hline Tumor maligno & $7(46,7 \%)$ & $40(31,0 \%)$ & $3(17,6 \%)$ \\
\hline Insuficiencia renal & $2(13,3 \%)$ & $37(28,7 \%)$ & $7(41,2 \%)$ \\
\hline Diabetes no insulino dependiente & $3(20 \%)$ & $41(31,8 \%)$ & $1(5,9 \%)$ \\
\hline Hiperplasia de la próstata & $5(33,3 \%)$ & $32(24,8 \%)$ & $6(35,3 \%)$ \\
\hline EPOC & $0(0 \%)$ & $16(12,4 \%)$ & $1(5,9 \%)$ \\
\hline Cardiomiopatía isquémica & $1(6,6 \%)$ & $13(10,1 \%)$ & $2(11,8 \%)$ \\
\hline Tumor maligno secundario de los huesos y de la medula ósea & $0(0 \%)$ & $13(10,1 \%)$ & $3(17,6 \%)$ \\
\hline Otros trastornos especificados de la próstata & $0(0 \%)$ & $10(7,8 \%)$ & $2(11,8 \%)$ \\
\hline \multicolumn{4}{|l|}{ Tratamientos recibidos } \\
\hline Agonistas/antagonistas LHRH", n (\%) & $14(93,3)$ & $122(94,6)$ & $16(94,1)$ \\
\hline Castración quirúrgica , n (\%) & $1(6,7)$ & $7(5,4)$ & $1(5,9)$ \\
\hline Bicalutamida, n (\%) & $6(40)$ & $55(42,6)$ & $11(64,7)$ \\
\hline Flutamida, n (\%) & $1(6,7)$ & $5(3,9)$ & $1(5,9)$ \\
\hline Docetaxel, n (\%) & $3(20)$ & $16(12,4)$ & $2(11,7)$ \\
\hline Cabazitaxel, n (\%) & $1(6,7)$ & $7(5,4)$ & $1(5,9)$ \\
\hline
\end{tabular}

*En 3 de los pacientes en este grupo no se pudo identificar la localización geográfica.

**10 principales comorbilidades (no se excluyen mutuamente - el mismo paciente puede haber tenido dos o más comorbilidades).

"se incluyeron los siguientes Agonistas/ antagonistas LHRH: leuprolida, goserelina, triptorelina y degarelix.

Incluye orquiectomía unilateral y bilateral.

Fuente: elaborado por los autores, 2019.

COP, siendo para Enzalutamida de $\$ 144.897,479$; y para acetato de abiraterona de $\$ 124.169,179$ (esos costos incluyen tanto el uso de servicios como el consumo de medicamentos). Los pacientes con terapia secuencial no alcanzaron los 12 meses de seguimiento, el costo de esa terapia a los 9 meses de seguimiento fue de $\$ 153.174,207,00$. Comparando los costos de los pacientes en las diferentes terapias, se evidenció que un paciente con terapia secuencial es el más costoso y tiene una menor sobrevida que con las otras terapias.
Al estimar los costos de los medicamentos, se obtuvo que 12 meses de tratamiento continuo con enzalutamida tuvieron un costo de \$136.911,489; mientras que el costo promedio con abiraterona fue $\$ 115.545,819$, por paciente. Sin embargo, el uso de servicios con esa última tecnología fue mayor durante el periodo de seguimiento. La - Figura 3 presenta los costos por categoría de servicio.

El gasto derivado del medicamento representa la mayor parte del total del costo del manejo del paciente con CPRCm, 
Tabla 2 Comorbilidades por grupos de edad

\begin{tabular}{|c|c|c|c|c|c|c|c|c|c|c|}
\hline Comorbilidad / grupo etario & $\begin{array}{l}50-59 \\
n=9\end{array}$ & Freq. & $\begin{array}{l}60-69 \\
n=32\end{array}$ & Freq. & $\begin{array}{l}70-79 \\
(N=70)\end{array}$ & Freq. & $\begin{array}{l}>80 \\
n=50\end{array}$ & Freq. & $\begin{array}{l}\text { Total } \\
(N=161)\end{array}$ & Freq. \\
\hline Hipertensión primaria & 3 & 0.33 & 25 & 0.78 & 52 & 0.74 & 35 & 0.7 & 115 & 0.71 \\
\hline Diabetes insulino dependiente & 4 & 0.44 & 12 & 0.37 & 23 & 0.32 & 14 & 0.28 & 53 & 0.33 \\
\hline Tumor maligno & 4 & 0.44 & 9 & 0.28 & 22 & 0.31 & 15 & 0.3 & 50 & 0.31 \\
\hline Insuficiencia renal & 1 & 0.11 & 3 & 0.09 & 24 & 0.34 & 15 & 0.3 & 43 & 0.27 \\
\hline Hiperplasia prostática & 4 & 0.44 & 9 & 0.28 & 18 & 0.25 & 12 & 0.24 & 43 & 0.18 \\
\hline Diabetes no insulino dependiente & 1 & 0.11 & 5 & 0.15 & 14 & 0.2 & 9 & 0.18 & 29 & 0.27 \\
\hline Enfermedad pulmonar obstructiva & 0 & 0 & 3 & 0.09 & 5 & 0.07 & 9 & 0.18 & 17 & 0.11 \\
\hline Cardiopatía isquemica & 2 & 0.22 & 1 & 0.03 & 9 & 0.12 & 4 & 0.08 & 16 & 0.10 \\
\hline $\begin{array}{l}\text { Tumor maligno secundario } \\
\text { de hueso y médula ósea }\end{array}$ & 0 & 0 & 3 & 0.09 & 10 & 0.14 & 3 & 0.06 & 16 & 0.10 \\
\hline $\begin{array}{l}\text { Otros trastornos } \\
\text { específicos de la próstata }\end{array}$ & 0 & 0 & 0 & 0 & 6 & 0.08 & 6 & 0.12 & 12 & 0.07 \\
\hline Enfermedad cardiovascular & 1 & 0.11 & 1 & 0.03 & 6 & 0.08 & 2 & 0.04 & 10 & 0.06 \\
\hline Angina inestable & 0 & 0 & 0 & 0.78 & 7 & 0.1 & 3 & 0.06 & 10 & 0.06 \\
\hline
\end{tabular}

Fuente: elaborado por los autores, 2019.

Tabla 3 Frecuencia de uso de servicios por paciente al mes

\begin{tabular}{|c|c|c|c|c|c|}
\hline Tipo de recurso & Media (DE) & Mediana & IC 95\% & Min & Max \\
\hline \multicolumn{6}{|l|}{ Consulta Externa } \\
\hline Enzalutamida $(n=15)$ & $1,74(1,08)$ & 1,73 & $1,15-2,34$ & 0,10 & 4,22 \\
\hline Acetato de Abiraterona $(n=129)$ & $1,61(1,50)$ & 1,67 & $1,35-1,87$ & 0,08 & 13,50 \\
\hline Terapia secuencial $(n=17)$ & $1,73(1,23)$ & 1,60 & $1,10-2,36$ & 0,50 & 4,67 \\
\hline \multicolumn{6}{|l|}{ Exámenes de Laboratorio } \\
\hline Enzalutamida $(n=15)$ & $3,37(4,06)$ & 1,58 & $1,12-5,62$ & 1,00 & 14,00 \\
\hline Acetato de Abiraterona $(n=129)$ & $3,34(5,31)$ & 2,92 & $2,41-4,26$ & 0,10 & 36,33 \\
\hline Terapia secuencial $(n=17)$ & $4,43(4,95)$ & 4,29 & $1,88-6,97$ & 0,17 & 16,50 \\
\hline \multicolumn{6}{|l|}{ Imágenes } \\
\hline Enzalutamida $(n=15)$ & $0,15(0,26)$ & 0,25 & $0-0,30$ & 0,09 & 0,89 \\
\hline Acetato de Abiraterona $(n=129)$ & $0,34(0,69)$ & 0,33 & $0,21-0,46$ & 0,08 & 1,13 \\
\hline Terapia secuencial $(n=17)$ & $0,24(0,40)$ & 0,37 & $0,03-0,44$ & 0,08 & 1,13 \\
\hline \multicolumn{6}{|c|}{ Consulta por Urgencias (pacientes no admitidos) } \\
\hline Enzalutamida $(n=15)$ & $0,02(0,03)$ & 0,11 & $0,01-0,04$ & 0,09 & 0,17 \\
\hline Acetato de Abiraterona $(n=129)$ & $0,15(0,42)$ & 0,18 & $0,08-0,23$ & 0,08 & 2,00 \\
\hline Terapia secuencial $(n=17)$ & $0,03(0,02)$ & 0,12 & $0,02-0,04$ & 0,08 & 0,14 \\
\hline \multicolumn{6}{|c|}{ Consulta por Urgencias (pacientes admitidos) } \\
\hline Enzalutamida $(n=15)$ & $0,06(0,09)$ & 0,26 & $0,01-0,11$ & 0,09 & 0,33 \\
\hline Acetato de Abiraterona $(n=129)$ & $0,07(0,14)$ & 0,08 & $0,04-0,09$ & 0,08 & 0,75 \\
\hline Terapia secuencial $(n=17)$ & $0,08(0,13)$ & 0,08 & $0,01-0,14$ & 0,08 & 0,42 \\
\hline \multicolumn{6}{|l|}{ Días de hospitalización } \\
\hline Enzalutamida $(n=15)$ & $4(3)$ & 6 & $2-6$ & 1 & 13 \\
\hline Acetato de Abiraterona $(n=129)$ & $6(7)$ & 7 & $5-7$ & 1 & 30 \\
\hline Terapia secuencial $(n=17)$ & $7(6)$ & 7 & $4-10$ & 1 & 27 \\
\hline
\end{tabular}

Fuente: elaborado por los autores, 2019. 


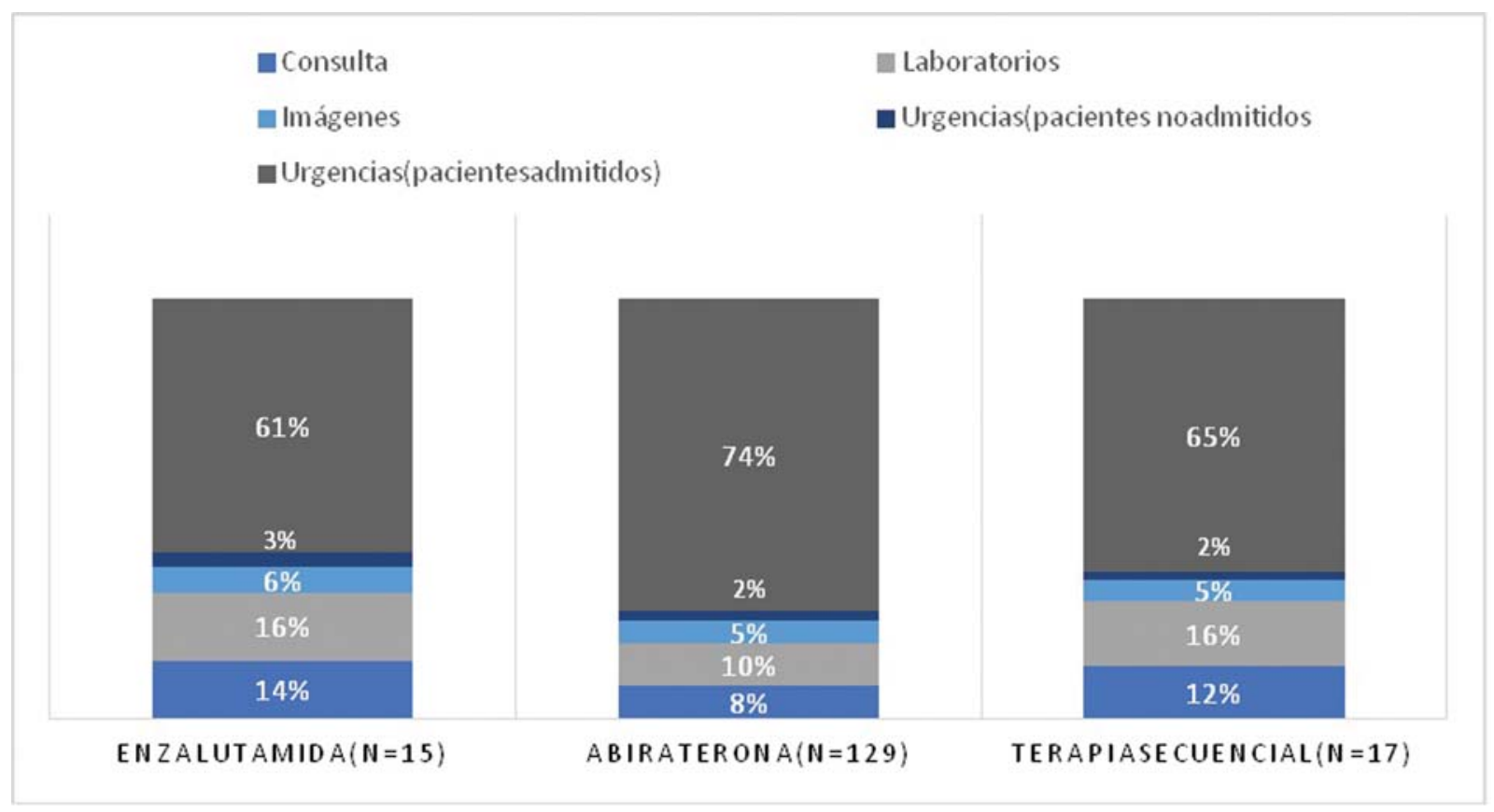

Fig. 3 Distribución de costos por categoría de servicio. Fuente: elaborado por los autores, 2019. Costo reportado en pesos colombianos (COP) 2016.

siendo 94,49\% para Enzalutamida; y el 93,06\% para el caso de abiraterona.

Como desenlace exploratorio se calculó la supervivencia a 10 años con el método del índice de comorbilidad de Charlson ${ }^{11}$,. Encontrando que la supervivencia a 10 años para esos pacientes es $0 \%$.

\section{Discusión}

Los resultados de este estudio indican que, aunque los pacientes tratados con enzalutamida tienen un mayor costo asociado al medicamento, los costos por uso de servicios de los pacientes con abiraterona son mayores, datos muy similares a lo encontrado en Estados Unidos, donde se evidencia que el costo de la tecnología de intervención (enzalutamida) es mayor al del costo de acetato de abiraterona, ${ }^{12,13}$ mientras que los costos asociados al uso de los servicios sanitarios, son menores en el grupo de pacientes con enzalutamida debido a que presentan menos hospitalizaciones, consultas por urgencias y exámenes que el grupo de acetato de abiraterona $^{12}$. Se concluye que el uso de la enzalutamida para este estadio del cáncer de próstata, resulta costoefectivo para el sistema de salud estadounidense. ${ }^{13}$ Un estudio japonés determinó que en el costo directo de atención de los pacientes con CPRCm la categoría medicamentos representa el $91 \%$, seguido de exámenes de laboratorio e imágenes con 4,8\%, 4,1\% para radioterapia y el $0,1 \%$ en cirugía. ${ }^{14}$ Un estudio llevado a cabo en Italia, estableció que los costos directos del manejo del CPRCm representan el $0,2 \%$ de la financiación del Servicio de Salud, siendo aproximadamente el $77 \%$ derivado de la dispensación de medicamentos. $^{15}$
Los datos de este estudio mostraron que el $43,5 \%$ de los pacientes están en el rango de edad de 70 a 80 años, así mismo el 74,6\% de los pacientes tenían 70 años, es decir que la enfermedad se puede asociar con edades avanzadas. En cuanto a la localización de los pacientes, se evidencio que en el departamento del Valle del Cauca encontramos la mayor proporción de pacientes. El 98,1\%, de los pacientes analizados pertenecían al régimen contributivo. Se debe tener en cuenta que esos datos provienen de una única EPS y que esas cifras pueden obedecer a las características de esa institución.

Se realizó el cálculo de la supervivencia a 10 años del paciente con el índice de comorbilidad de Charlson y, a lo largo de los diferentes rangos de edad, se demostró que los pacientes tenían una tasa de supervivencia del $0 \%$ a los 10 años. En nuestra población evaluada, las comorbilidades más frecuentes encontradas fueron hipertensión esencial, diabetes mellitus, insuficiencia renal y otras patologías tumorales relacionadas con el cáncer de próstata.

Este es el primer análisis de costos de pacientes con cáncer de próstata metastásico resistente a la castración $(\mathrm{CPRCm})$ desarrollado con datos de la atención real en Colombia. Los datos muestran que los pacientes que usan abiraterona tienen un mayor uso de recursos en salud que los pacientes tratados con enzalutamida, encontrando que para los servicios de imágenes el costo es $73 \%$ más para los pacientes con abiraterona, así mismo el costo de los pacientes admitidos (hospitalizados) es del $148 \%$ más con abiraterona.

Dentro de las limitaciones de este estudio, están las relacionadas con el desarrollo de estudios de tipo transversal ya que están basados en la información que proviene de las bases de datos y eso puede generar vacíos 
de información, sin embargo, la validación de los datos encontrados por parte de expertos clínicos hace que se puedan reducir los sesgos en el análisis por estadio de la enfermedad y uso de servicios asociado a la patología.

En este análisis, la población total incluyó 161 pacientes, $80 \%$ tratados con Abiraterona y 9\% tratados con Enzalutamida, se observa que la diferencia entre los grupos es bastante amplia, debido a que el ingreso de enzalutamida al mercado colombiano fue en junio de 2016, un mes antes de los datos tomados para el análisis. Los datos presentados en este análisis, permiten evaluar el manejo del paciente con la enfermedad en cuanto a uso de recursos, uso de tecnologías y beneficios en la atención relacionados con los modelos de atención del paciente en estadio metastásico resistente a castración en cáncer de próstata.

Este estudio muestra como los pacientes con la enfermedad requieren recursos de salud constantemente, relacionados con imágenes, laboratorios, hospitalizaciones y medicamentos siendo ese último ítem, el de mayor peso en el costo total.

Al comparar la utilización de servicios sanitarios entre ambas tecnologías, se muestra que los pacientes tratados con enzalutamida requirieron menos imágenes, visitas a la sala de emergencias (pacientes no ingresados) y, para las visitas a las salas de emergencias (pacientes ingresados), la estadía fue más corta que la de los pacientes tratados con abiraterona.

Por otro lado, los pacientes tratados con terapia secuencial, fueron tratados solo con acetato de abiraterona en primer lugar, pero luego, cuando la enzalutamida estuvo disponible en el mercado, la terapia se cambió de acetato de abiraterona a enzalutamida en el $88,2 \%(n=15)$ del total de pacientes.

El presente análisis mostró que el costo de un paciente con CPRCm depende tanto de la molécula seleccionada como del uso de recursos de salud. Ese último determinado, a su vez, por diversos factores como podrían ser las características de la propia patología, requerimientos de seguimiento $\mathrm{y}$, por los eventos adversos y complicaciones asociados a la molécula. Se necesitan más estudios que comparen la utilización de servicios sanitarios y el costo entre la enzalutamida y la abiraterona para respaldar los hallazgos presentados.

Financiamiento

El presente estudio fue financiado por Astellas Farma Colombia S.A.S.

\section{Referencias}

1 Taitt HE. Global Trends and Prostate Cancer: A Review of Incidence, Detection, and Mortality as Influenced by Race, Ethnicity, and Geographic Location. 2018

2 Cuenta de alto costo. Situación del Cáncer en la población adulta atendida en el SGSSS de Colombia 2016. Bogotá2016

3 American cancer society. Cancer de prostata [Internet]. 2020. Available from: https://www.cancer.org/es/cancer/cancer-deprostata.html

4 Moro Soria A, Laborí Cardá C, Bouzó López A, González Hernández J. El cáncer de próstata resistente a castración. Mecanismos de progresión y nuevos tratamientos. Rev Cuba Urol [Internet] 2012; 1(01):106-122. Available from: http://www.revurologia.sld.cu/ index.php/rcu/article/view/15/18

5 National Comprehensive Cancer Network. Prostate CancerVersion 1.2020. NCCN Clin Pract Guidel Oncol [Internet] 2020:938-944. Available from: https://www.nccn.org/professionals/physician_gls/ default.aspx

6 Asociación española de urología. Protocolo de actuación en Pacientes con Cáncer de Próstata resistente a la castración [Internet]. 2014. Available from: https://aprenderly.com/doc/1111306/protocolo-deactuación-en-pacientes-con-cáncer-de-próstata

7 European Medicines Agency (EMA). FICHA TÉCNICA O RESUMEN DE LAS CARACTERÍSTICAS DEL PRODUCTO - Acetato de abiraterona (ZYTIGA) [Internet]. 2016. Available from: https://www.ema. europa.eu/en/documents/product-information/zytiga-epar-productinformation_es.pdf

8 Ministerio de salud y protección social. PosPopuli [Internet]. 2020. Available from: https://pospopuli.minsalud.gov.co/Pospo puliWeb/paginas/home.aspx

9 IETS. Análisis de impacto presupuestal de hormonoterapia para el tratamiento de cáncer de próstata en Colombia [Internet]. Bogotá2017. Available from: https://www.iets.org.co/Archivos/ 27/Hormonoterapia_12-12-17.pdf

10 PAHO. Actualizaciones de la CIE-10 [Internet]. Available from: https:// www.paho.org/hq/index.php?option=com_content\&view=article\& id=9178:2013-actualizaciones-cie-10\&ltemid=40350\&lang=en

11 Charlson ME, Pompei P. Ales KL. a New Method of Classifying Prognostic in Longitudinal Studies: Development. J Chronic Dis 1987;40(05):373-383https://pubmed.ncbi.nlm.nih.gov/ 3558716/ [Internet]

12 Kassabian, et al. Health care resource utilitization and costs in metastatic castration-resistant prostate cancer patients treated with enzalutamide or abiraterone acetate. Atlanta2018

13 Barqawi YK. Cost Effectiveness Analysis of Enzalutamide, Abiraterone Plus Prednisone and Cabazitaxel Plus Predinsone for the Treatment of Visceral Metastatic Castration Resistant Prostate Cancer (MCRPC) after Docetaxel Therapy. 2018

14 Satoh T, Ledesma D, Yoshihara N. The Economic Burden of Metastatic Castration Resistant Prostate Cancer and Skeletal Related Events in Japanese University Hospitals. Asian Pac J Cancer Prev 2018;19(01):21-26

15 Restelli U, Ceresoli GL, Croce D, et al. Economic burden of the management of metastatic castrate-resistant prostate cancer in Italy: a cost of illness study. Cancer Manag Res 2017;9:789-800 